\title{
DESIGN AND FABRICATION OF PLANETARY DRIVE MAGNET PEDAL POWER HUB-DYNAMO
}

\author{
Vivek Kumar ${ }^{1}$, Dr. Prakash Verma ${ }^{2}$ \\ ${ }^{\mathbf{1}}$ Research scholar (Machine Design), ${ }^{\mathbf{2}}$ Professor, \\ ${ }^{1,2}$ Department of Mechanical Engineering, Jabalpur Engineering College, Jabalpur, India.
}

\begin{abstract}
:
This paper presents a low speed permanent magnetic based generator which is suitable for supplying generating power from bicycle motion and application in providing energy for bicycle front and rear lights or electronics devices.The dynamo have a hub axel, a hub housing rotatable mounted around the hub axel with bearing, a planetary drive that increases the rotational speed of the permanent magnet, and the power generating mechanism with coil fitted to hub axel that has connected to the output connector. In such a hub dynamo, the magnet rotates faster than the bicycle wheel so that power output is high even at the normal bicycle speeds.
\end{abstract}

\section{Keywords:}

Pedal power, Hub-dynamo, planetary drive, Creo 2.0

\section{INTRODUCTION:}

The hub generator have been popular since the 1950s as bottle dynamo and stationary bicycle dynamo. Standard bottle generators running on the tire tread or rim suffer from frictional losses at the wheel/generator interface that also increases the heat at tire/generator interface. This type of generators are unreliable in wet weather (Khandewal et al, 2014). Bicycle hub generator offers a simple and reliable way to generate power for bicycle front and rear lights. But only in recent years have efficient generator hubs become available that generate about $2.4 \mathrm{~W}$ power and moderate bicycle speed (N Brito et al, 2006). The device described in this paper integrates power higher than these dynamo and allowing the generation of environment-friendly electric power while keeping fit by pedaling. This contemplates both personal health and environmental issues (M N haji et al, 2010). With increasing concern about global warming, greenhouse gas emission $\&$ rising fuel prices, non-motorized modals, such as bicycle aregaining importance as viable mode in urban transportation worldwide (Majumdar et al, 2014).A dynamo is coupled to bicycle. This arrangement enables the lighting of front, rear light of bicycle and charging of mobile phone, if a cyclist pedals fast enough. The device is very suitable for the village and those areas where electricity is not available. Moreover, it can be a completely autonomous energy source without the need for maintenance. Taking in to account the available volume inside a bicycle hub and that the rotation due to bicycle movement are possibly the best energy source in this case, a multi polar magnet and armature was considered as the best way to harvest energy to power the bicycle electronic devices. In the last decade much research has been done in order to develop devices that harvest energy from applications environmental and it into electrical energy (A R mech. et al, 2012). This work is directed to bicycles and, more particularly, to the multi-pole dynamo having a stack that is formed by plate-shaped members. The stack of multi-pole dynamo is formed using pressed electrical steel sheet (Takahiro Endo et al, 2003). A dynamo has a multi stack armature with coil fixed to a hub axel and a magnet casing that can rotate relatively higher speed than the 
bicycle wheel speed. The casing has transfer power by planetary drive to magnet holder. The armature stack that houses the coil comprises multiple laminated thin plate-shaped members to prevent the generation of eddy currents.

\section{Design Concept and Principle of Operation}

\subsection{Design Concept of hub-dynamo:}

Figure 1 illustrates the design scheme of the hub-dynamo proposed in this study. The precise 3D modal of hub dynamo was prepared by Creo 2.0 software as per Indian bicycle standards. A hubdynamo is mounted to distal ends of right and left forks of a front wheel of a bicycle. A hub dynamo comprises a hub shaft structured to be mounted at the bottom end of front fork, a hub shell structured to rotate around hub shaft, a rotating member in the form of a driven body disposed radially inwardly of hub shell and structured to rotate around hub shaft, a planetary gear transmission that transmits rotation of hub shell to driven body. A power generating mechanism is disposed between driven body and hub shaft.Both end of the hub shaft are fixed to front fork. A portion of large diameter portion forms a sun gear of planetary gear transmission. Hub shell is a substantially cylindrical member formed in two pieces, and it is rotatable supported on hub shaft at both ends by bearing assemblies Casing has a pair of hub flanges, and multiple mounting holes on the flanges for mounting the ends of the wheel spokes are formed at equal intervals along the circumferences of the respective hub flanges.

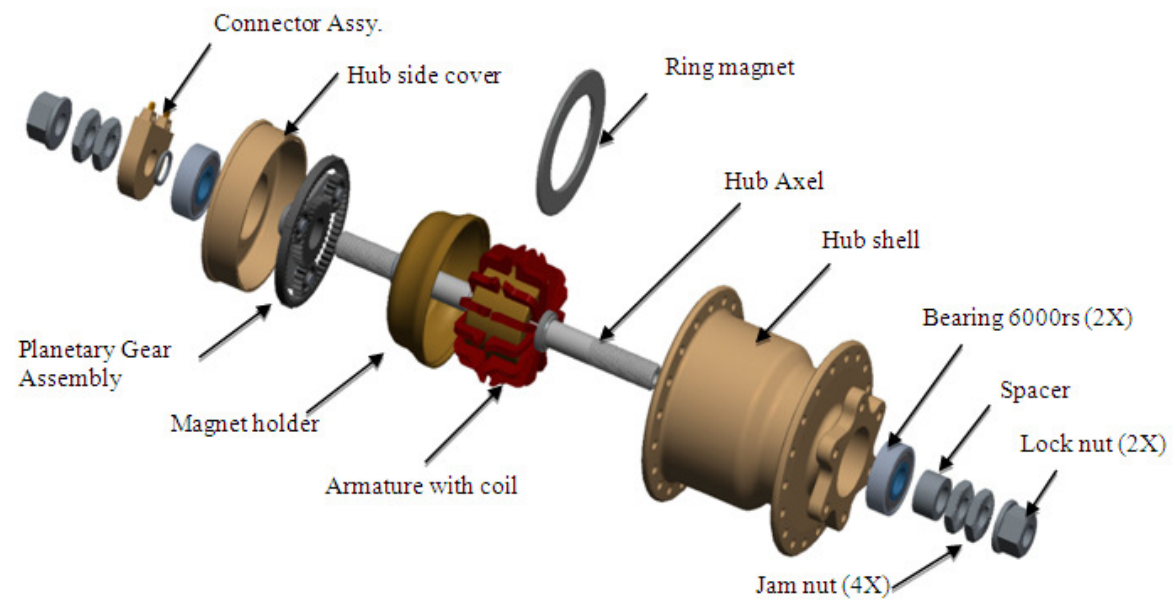

Figure. 1 Exploded view of Hub dynamo

Planetary gear transmission includes sun gear formed on hub shaft, ring gear formed on the inner peripheral surface of hub side cover, a planet gear carrier stationary supported by large-diameter portion of hub shaft, and a plurality of (e.g., three) planet gear supported by planet gear carrier. Planet gear carrier is very stable during operation and does not tilt. Planetary gear transmission communicates increased rotation of hub shell to driven body. The rotation of sun gear of planetary gear transmission can be expressed by the following expression:

$$
\mathrm{Ns}=(\mathrm{Zr} / \mathrm{Zs}) \times \mathrm{Nr}
$$

Where $\mathrm{Nr}$ and $\mathrm{Zr}$ is the rotation and number of teeth on ring gear and $\mathrm{Ns}$ and $\mathrm{Zs}$ is the rotation number of teeth on sun gear. When the number of teeth $\mathrm{Zs}$ of sun gear is set at $32 \mathrm{~T}$, and the number of teeth $\mathrm{Zr}$ of ring gear is set at $66 \mathrm{~T}$, then 


$$
\mathrm{Ns}=(66 / 32) \times \mathrm{Nr}=2.0625 \mathrm{Nr}
$$

As noted previously, power generating mechanism is disposed between driven body and hub shaft for generating power in response to relative rotation between driven body and hub shaft. Power generating mechanism comprises a magnet and a fixed unit, wherein magnet is fitted to the inner peripheral surface of driven body for rotation there with, and fixed unit is fixed to hub shaft. In this design, a ring-shaped magnet comprises 13 set of alternating N-poles and S-poles spaced apart in the circumferential direction of driven body. The interior fixed armature has a ringshaped coil and a stack assembly that is disposed such that it surrounds the coil. The stack assembly and coil are secured to the hub shaft such that they are sandwiched by a pair of nuts and are fastened to screw members formed on the outer circumferential surface of the hub shaft. A cord passing groove is formed on the outer peripheral surface of hub shaft to accommodate connecting cord that is connected to coil and communicates the generated power to an output connector mounted to hub shaft outside of hub shell. Electric wiring communicates the generated power from connector to switching unit.

The complete specification of the generator is given in Table 1 .

Table 1.

Hub-dynamo specifications

\begin{tabular}{ll}
\hline Parameters & \\
\hline Voltage & $6 \mathrm{~V}$ \\
Output & $3 \mathrm{~W}$ \\
Brake Type & Disc Brake \\
Hub shell Material & 6061 \\
& Aluminum \\
Bearing type & Sealed cartridge \\
Hub Axle Diameter & $10 \mathrm{~mm}$ \\
Hub axle length & $140 \mathrm{~mm}$ \\
Over locknut dimension & $100 \mathrm{~mm}$ \\
Spokes holes & 36 \\
Pitch circle diameter & $72 \mathrm{~mm}$ \\
Flange width & $57 \mathrm{~mm}$ \\
Weight & $800 \mathrm{grams}$ \\
\hline
\end{tabular}

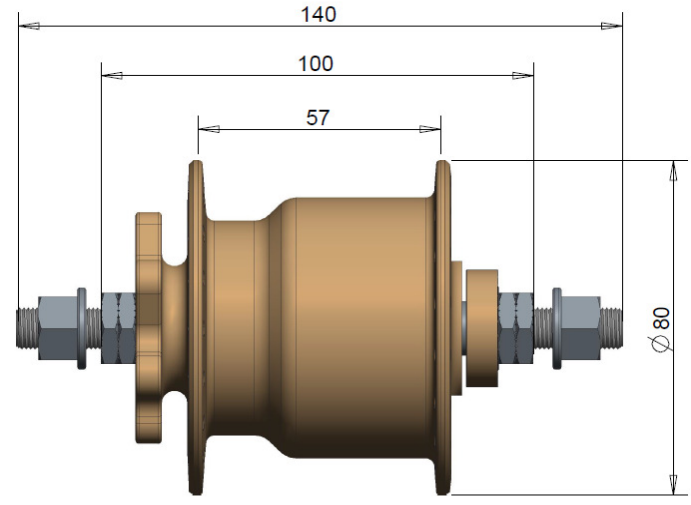

Figure. 2 Hub dynamo 


\subsection{Principal Operation of Hub dynamo:}

When the front wheel, i.e. the hub casing, rotates relative to the hub axel as the bicycle travels forward, the planetary drive rotates the permanent multi pole magnet around the coil. When the vertical outer portions of the plate shaped pieces forming the first stack arm receive N-pole magnetic flux from the multi pole permanent magnet, then the radially outer portion of the plateshaped pieces forming the second stack receive S-pole magnetic flux. Conversely, when the radially outer portion of the plate-shaped pieces forming the stack receive S-pole magnetic flux from the permanent magnet, then the radially outer portion of the plate-shaped pieces forming the second stack receive $\mathrm{N}$-pole magnetic flux.

By virtue of the rotation of the permanent magnet around the radially outer portions of the plateshaped pieces forming the first and second stack the hub dynamo repeated alternates between a first state in which the first stack comprise the N-pole and the second stack comprise the S-pole. As a result, alternating magnetic flux occurs in the radially inner portion of the magnetically linked stack of plate-shaped pieces. The alternating magnetic flux produces inside the coil and current flow through the coil. The generated power transfer by the wire to the connector and connector connect the all electrical devices of the bicycle.

\section{Experiment results and discussion:}

The apparatus mounts the hub shell in a fork and turns it by a dc motor speed is measured in rpm by a digital meter. As per the standard JIS.c.9502, the electrical output is monitored with $12 \mathrm{ohm}$ and $15 \mathrm{ohm}$ resistances, science that's how it's always been done, as bulbs are also resistors. Output of the hub dynamo is measured by a multimeter with open circuit, $12 \mathrm{ohm}$ and $15 \mathrm{ohm}$ resistances.

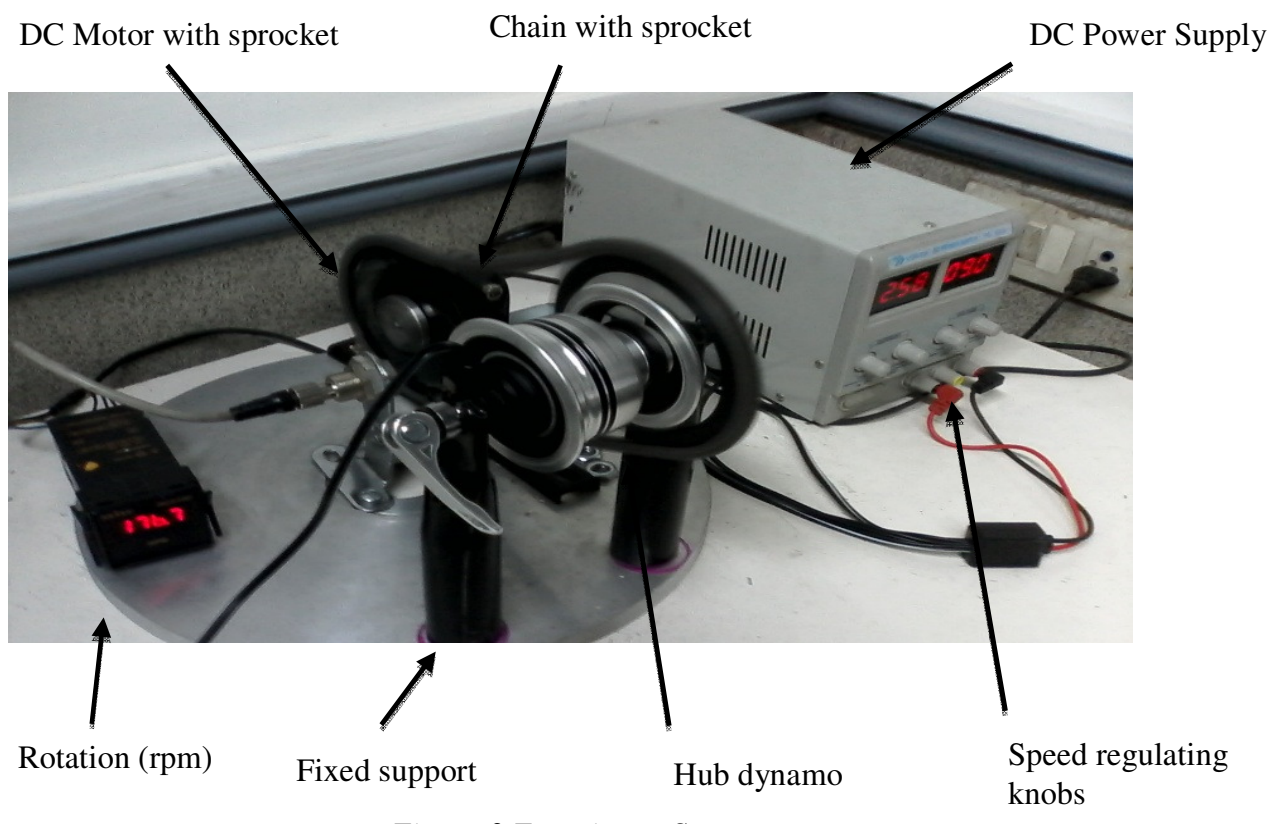

Figure.3 Experiment Setup 
Advances in Materials Science and Engineering: An International Journal (MSEJ), Vol. 2, No. 3, September 2015

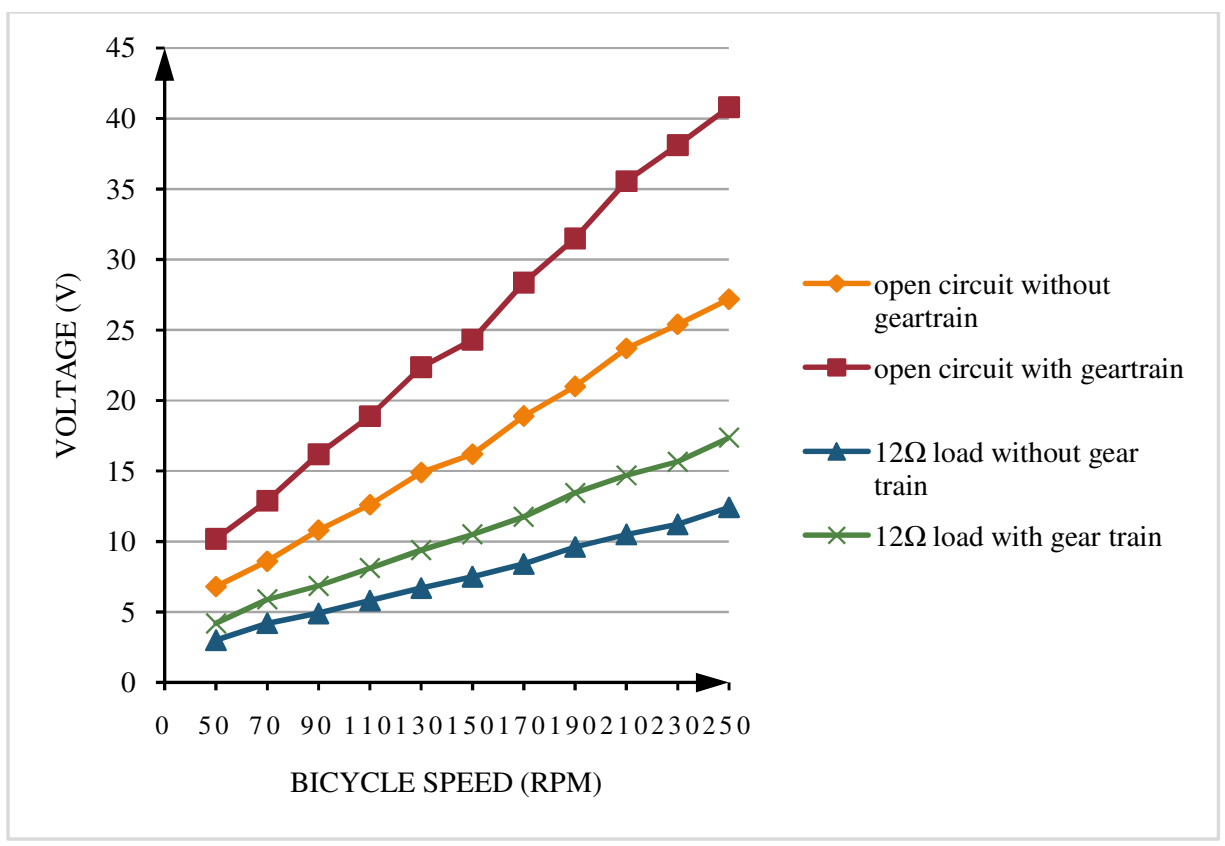

Figure.4 Voltage output over Bicycle speed according to test and calculation

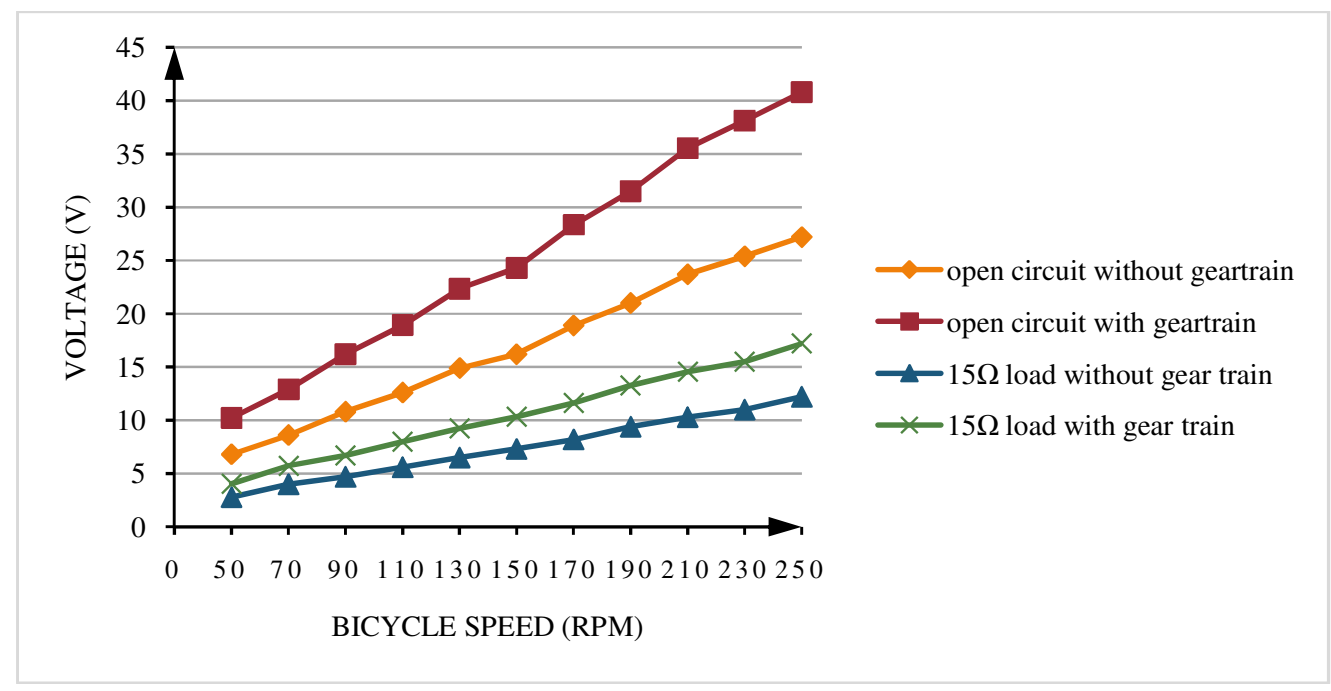

Figure.5 Voltage output over Bicycle speed according to test and calculation

Figure $4 \& 5$ depicts the calculated and measured voltage output versus bicycle speed for the biologic hub dynamo and geared hub dynamo. In open circuit voltage measured voltage is without any resistance and also measured voltage at 12 and $15 \mathrm{ohm}$ resistances with geared and non-geared hub dynamo. It has seen that the voltage output of geared hub dynamo generate sufficient voltage for lightening the bicycle at normal speed $(8-12 \mathrm{~km} / \mathrm{h})$. The corresponding dynamo output power is depicted in figure $6 \& 7$. The power output over the bicycle speed is calculated by the measured voltage. The hub dynamo rotation also depend upon the wheel diameter, if the wheel diameter is less it takes more turn for a specific speed then the large diameter bicycle wheel. It can be seen, that planetary gear transmission should be preferred for 
low bicycle speed and the power output of hub dynamo is about 2.4-3 watt at normal bicycle speed $(8-12 \mathrm{~km} / \mathrm{h})$.

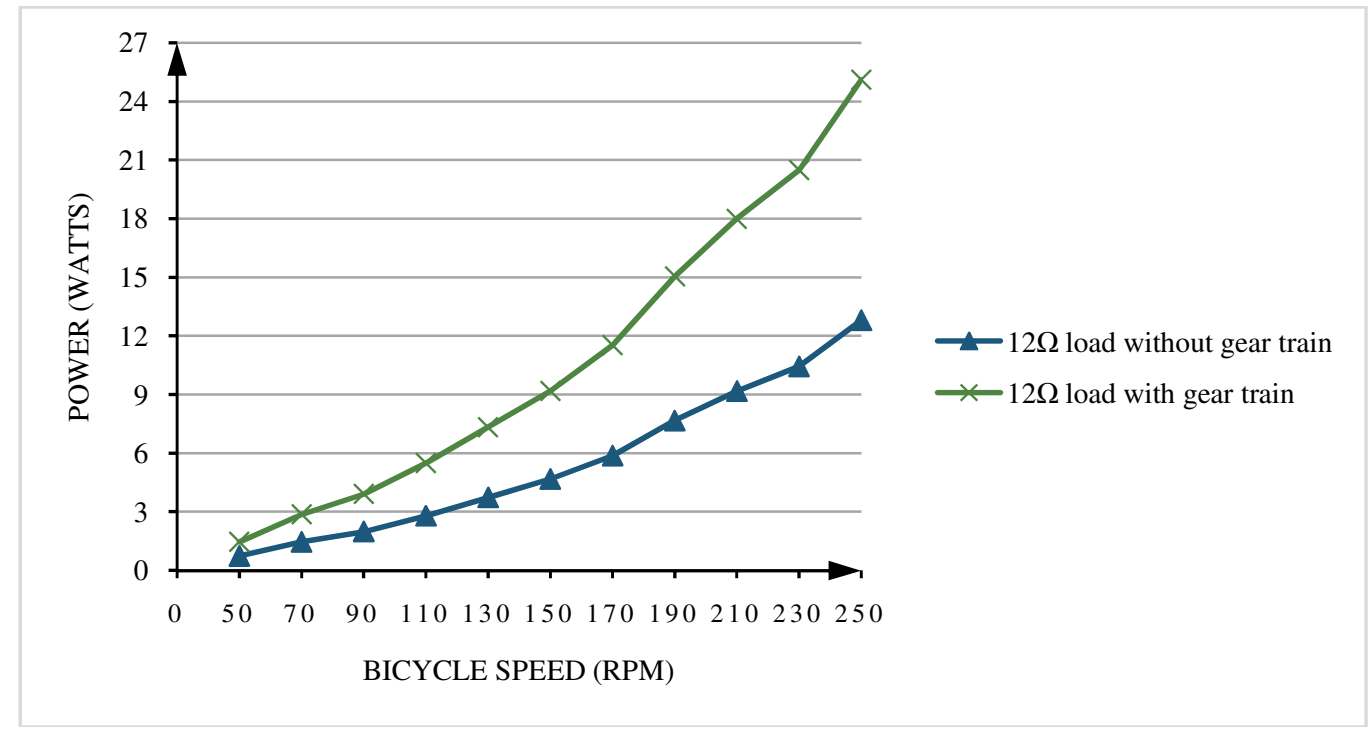

Figure.6 Power output over Bicycle speed according to test and calculation

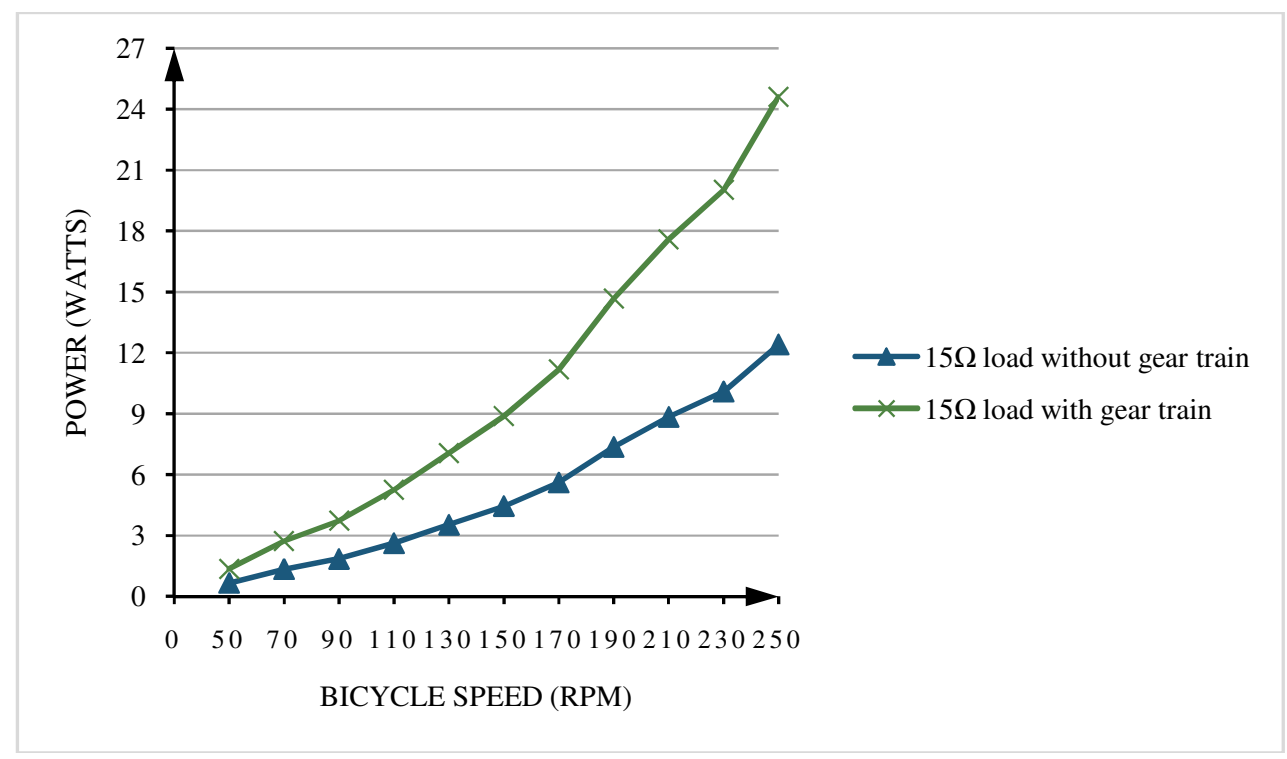

Figure.7 Power output over Bicycle speed according to test and calculation

\section{Conclusion:}

Planetary gear train is one of the key component of the Hub dynamo to increase the rotation at armature end. This paper presented a new design for a low speed permanent magnetic generator. The idea behind this study was to develop a suitable electricity generatorfor bicycle, which could power supply to bicycle lights and other electronic devices. This paper introduce the structure of a permanent magnet hub dynamo which could beneficial over the bottle dynamo and other type of 
bicycle dynamo. The prototype generators generated 2.4-3W power at the normal bicycle speed. These results indicates that the generated power by the bicycle hub generator is sufficient amount for lightening 2 to 3 watts load from the bicycle motion.

\section{REFERENCES:}

[1] R. Suhalka, M C Khandelwal, K K Sharma, A. Sanghi (June, 2014) "Generation of Electrical Power using Bicycle Pedal" International Journal of Recent Research and Review, Vol.VII, Issue 2.

[2] NunoBrito, Luis Ribeiro, Joao SenaEsteves (2006) "Electric Power Generating Bicycle" 3rd International Conference on Hands-on Science, H-Sci ISBN 9899509507.

[3] Maha N. Haji, Kimberly Lau, and Alice M. Agogino (2010) "Human Power Generation in Fitness Facilities," Proceedings of ASME 2010 4th International Conference on Energy Sustainability ES2010.

[4] S K Chandrakar, D L Yadav, C Kurre (2014) "Experimental study of micro industry of animal powered mechanical device for battery charging" $2^{\text {nd }}$ International Conference on Innovations in Automation and Mechatronics Engineering, ICIAME 2014.

[5] Cyders, T. and Kremer, G. G., (2008) "Engineering Around the World: Driving Local Economics in Africa with Human Power," Proceedings of the ASME 2008 International Mechanical Engineering Congress and Exposition (IMECE2008), Vol. 9: Engineering Education and Professional Development, pp. 181-187.

[6] C.R. Saha, T. O'Donnell, N.Wang, P. McCloskey (2008) "Electromagnetic generator for harvesting energy from human motion" Sensors and ActuatorsA 147, 248-253.

[7] Jansen, A. and Stevels, A.,(2006) "Combining eco-design and user benefits from human powered energy systems, a winwin,”Journal of Cleaner Production, 14 (15-16), pp. 1299-1306.

[8] Japanese Industrial Standard, JIS C 9502: 2008 (E), Lighting equipment for bicycles.

[9] Japanese Industrial Standard, JIS D 9419: 1996 Bicycle hubs.

[10] Indian Standard, IS 629 (1988): Bicycle Hub.

[11] United States Patent, Takeda et al.US007002280B2, Claw-Pole Dynamo With Radially Offset Yoke Arms.

[12] United States Patent, Liao et al.US 20100233914Al, Electrical Connector For A Dynamo Hub.

\section{Authors:}

Vivek Kumar Tiwari received his BE in Mechanical Engineering from RGPV, Bhopal in 2012 and perusing Masters in Engineering in Machine Design from Jabalpur Engineering College, Jabalpur. His research interest includes design, modelling and fabrication of machine components.

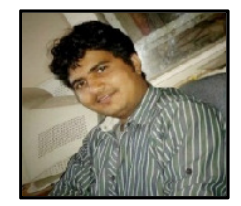

